\title{
Factors Influencing Hearing Disability and Reduction in Disability among First-time Hearing Aid Users in Singapore
}

Eu Chin $\underline{\text { Ho }},{ }^{1}$ DOHNS (RCSEng), MMedEdu (Warwick), FRCS (ORL-HNS), KeXin Li,${ }^{2}$ Warren Ming Wu Ong, ${ }^{2}$ Yen Tze Eileen Bei, ${ }^{1}$ Aruni Seneviratna ${ }^{1}$

\begin{abstract}
Introduction: This study aims to examine the factors associated with self-reported hearing disability and early reduction in disability after first-time hearing aid (HA) fitting in Singapore.

Methods: Retrospective record review of 1,068 subjects issued with HAs at a tertiary hospital from 2001 to 2013.

Results: Subjects reporting $\geq 5$ disabilities reduced from $90 \%$ to $24 \%$ after HA fitting. 'Difficulty hearing in noise' was the commonest disability before and after HA fitting, while 'needs to increase volume of TV/radio' was the disability with most improvement after fitting. In multivariable models, having worse pure tone audiometry (PTA) thresholds of the better hearing ear and being ethnically Chinese were associated with subjects reporting more hearing disabilities. A higher proportion of subjects reported a reduction rather than an absence of disability after HA fitting. In multivariable models, daily HA usage for $\geq 4$ hours, sensorineural hearing loss (HL) and worse PTA thresholds of the better hearing ear were associated with reduction in more disabilities after HA fitting.

Conclusion: Hearing disability is high among first-time HA users in Singapore. Ethnicity and PTA thresholds were associated with self-reported hearing disability. After HA fitting, higher daily HA usage, sensorineural HL, and worse PTA thresholds of the better hearing ear were associated with early reduction in disability. Patient counselling on the benefits of HL rehabilitation could focus on hearing disability rather than PTA thresholds. The management of patients' expectations could focus on reducing rather than eliminating disability.
\end{abstract}

Ann Acad Med Singap 2020;49:978-89

Keywords: Epidemiology, hearing loss rehabilitation, quality of life, sensorineural hearing loss, uptake

\section{Introduction}

The World Health Organization estimates that over 5\% of the world's population suffers from disabling hearing loss (HL), defined as better hearing ear pure tone audiometry (PTA) thresholds of $>40 \mathrm{~dB} .{ }^{1}$ The 2016 Global Burden of Disease study ranked HL as the third leading cause of years lived with disability globally. ${ }^{2}$

Impairment refers to the reduction in function of an organ while disability describes its impact on day-today activities. Handicap refers to the disadvantages encountered by individuals in fulfilling their normal roles. ${ }^{3}$ Untreated hearing impairment can lead to hearing disability like communication and sound localisation difficulties. Hearing handicap includes education disadvantage, ${ }^{4}$ under-employment and unemployment, ${ }^{5,6}$ and impaired social relationships. ${ }^{7-9}$ Hearing aid (HA) use is associated with improved social functioning and employment opportunities, ${ }^{6}$ improved $\operatorname{cognition}^{10}$ and lower depression risks. ${ }^{11}$

Self-reported hearing disabilities may be better than PTA thresholds in predicting HA uptake, ${ }^{12,13}$ use, satisfaction ${ }^{10,14}$ and benefits. ${ }^{15}$ In spite of Singapore's

\footnotetext{
${ }^{1}$ Department of Otorhinolaryngology, Tan Tock Seng Hospital, Singapore

${ }^{2}$ NUS Yong Loo Lin School of Medicine, National University of Singapore, Singapore

Address for Correspondence: Dr Eu Chin Ho, Department of Otorhinolaryngology, Tan Tock Seng Hospital, 11 Jalan Tan Tock Seng, Singapore 308433.

Email: euchinho@yahoo.co.uk
} 
standing as a high income nation, ${ }^{16}$ the 2010 Singapore National Health survey found that only $3.3 \%$ of adults with disabling HL use HAs. ${ }^{17}$ This compares unfavourably with other developed countries: $14.1 \%$ in Japan, ${ }^{18} 14.3 \%$ in the US, ${ }^{19} 18.4 \%$ in Taiwan, ${ }^{20}$ and $38.6 \%$ in the UK. ${ }^{21}$ Singaporeans seek help only when HL is advanced. ${ }^{22}$ Only $36 \%$ of HA users reported daily usage of $>7$ hours, ${ }^{22}$ compared to the UK (52), ${ }^{21}$ Switzerland $(57 \%)^{23}$ and Germany $(58 \%){ }^{24}$

Evaluation of the benefits of HA is challenging. A review comparing speech tests with patient questionnaires concluded that speech tests may not represent benefits experienced in a real-world listening environment. ${ }^{25}$ Findings from questionnaires, such as the commonly used Hearing Handicap Inventory for the Elderly (HHIE) before and after HA fitting may be limited by errors in captured changes in benefits. ${ }^{25}$

This study was performed to answer the following questions: (1) what are the hearing disabilities and how severe are they in Singapore; (2) to what extent does HA usage reduce hearing disability; and (3) what are the factors associated with hearing disability and reduction in hearing disability.

\section{Methods}

\section{Design}

A retrospective record review of consecutive first-time HA users at Tan Tock Seng Hospital in Singapore between 2001 and 2013 was performed.

\section{Setting}

Patients with suspected hearing impairment underwent a diagnostic PTA. Those motivated to improve their hearing were given a hearing aid evaluation (HAE) appointment, followed by a hearing aid fitting appointment, and a post-hearing aid fitting (PHAF) appointment at least 1 month later, carried out by audiologists using standardised protocols.

The Client Orientated Scale of Improvement (COSI), which allows patients to nominate 5 listening situations in which they need help, was shown to be as accurate as the traditional and longer questionnaires in quantifying hearing disability. ${ }^{25}$ Using the COSI methodology, a list of the 8 commonest self-reported hearing disabilities was compiled, facilitating the identification, quantification and management of each patient's hearing disability profile. These questions were routinely administered before HAE and after HA fitting (PHAF), facilitating the evaluation of early changes in disabilities.

Questions 1 to 7 (Table 1) rated the level of difficulty with conversation, hearing and function as 'always', 'sometimes' or 'never'. For question 8, 'hearing loss limits social life', the level of difficulty was indicated as 'severe', 'moderate', 'slightly' or 'never'. This response scale captures the level of individual disability in finer granularity in comparison to a binary 'yes/no' response. However, all 'never' answers were delineated as 'no' and all other answers were delineated as 'yes' to facilitate data analyses for hearing disability before and after HA fitting (Table 1).

Subjects who chose not to answer were categorised as 'non-responders' for the corresponding question. Subjects had the option of answering 'not applicable' to 'hearing loss affects job' and 'family member feels frustrated' (questions 6 and 7).

When analysing the change in hearing disability, a reduction was defined as a change in response on the scale towards improvement at PHAF (e.g. 'always' to 'sometimes', or 'moderate' to 'slightly'). Absence of a reduction was defined as no change at PHAF or changes on the scale towards deterioration.

HL was determined by the 4 tone average hearing thresholds at $0.5,1,2$ and $4 \mathrm{kHz}$ tested independently for each ear. This study was approved by the Institutional Review Board (2013/00325). Waiver of consent was granted as data were anonymised and aggregated for analyses.

\section{Statistical analysis}

Statistical analyses were performed using Stata (version 13.1, StataCorp LP, College Station, US). Significance tests were two-sided at the $5 \%$ significance level. Categorical data were compared using the chi-square test or Fisher's exact test. Continuous data were compared using the t-test and analysis of variance (ANOVA, normally distributed) or the Mann-Whitney U test and Kruskal-Wallis test (skewed). Results were reported as count (n) and percentage by category for categorical data; mean, standard deviation (SD) or median; and interquartile range (IQR) for continuous data. Bonferroni correction was done for multiple comparisons for each type of difficulty at baseline and follow-up. Factors associated with hearing disability and reduction in disability were evaluated using logistic regression models. The crude odds ratio (OR) and adjusted odds ratio (AOR) are reported with the $95 \%$ confidence interval $(\mathrm{CI})$.

\section{Results}

Of the 1,068 subjects, $92.3 \%$ responded to at least 1 question at HAE (baseline), $73.7 \%$ responded to at least 1 question at PHAF (follow-up), and $71.3 \%$ had at least 1 valid response for both. The median time from 
HAE to PHAF appointment was 2 months (IQR 1 month). For the cohort with mean age of 70 years, $78 \%$ of subjects responded 'not applicable' to 'hearing loss affects job' (Singapore's retirement age is 62 years old).

The commonest disabilities where deterioration was reported were 'difficulty hearing in noise' (98.6\%), 'difficulty with group conversation in quiet' $(97.4 \%)$, and 'needs to increase volume of TV/radio' (95.1\%). The commonest residual disabilities at PHAF were 'difficulty hearing in noise' $(67.5 \%)$, 'difficulty hearing over the telephone' $(47.4 \%)$, and 'difficulty with group conversation in quiet' (44.9\%) (Table 1).

The commonest disabilities with reported reduction were 'needs to increase volume of TV/radio' $(84.0 \%)$, 'difficulty with group conversation in quiet' $(81.7 \%)$ and 'difficulty with 1:1 conversation' (78.9\%), while the least reported was 'family member feels frustrated' $(62.0 \%)$. Subjects who reported a reduction in disability included those with or without any residual disability.

Baseline disability was high with $90.2 \%$ reporting $\geq 5$ disabilities and $58.5 \%$ reporting $\geq 7$ disabilities. After HA fitting, the proportion dropped to $24.0 \%$ and $6.6 \%$, respectively. Conversely, only $1.7 \%$ of subjects had $\leq 2$ disabilities at baseline but this increased to $53.1 \%$ after HA fitting.

The commonest disabilities where deterioration was reported were 'difficulty hearing over the telephone' $(5.7 \%)$, followed by 'hearing loss limits social life' $(4.9 \%)$ and 'family member feels frustrated' (3.9\%).

\section{Comparison of responders and non-responders}

Of the 1,068 subjects, $79.1 \%$ responded to all 8 questions at HAE, and $64.3 \%$ responded to all 8 questions at both HAE and PHAF. Responders and nonresponders were compared to assess for any responder bias. Non-response was not question-specific and ranged from $8.1-12.6 \%$ for $\mathrm{HAE}$, and $27.3-30.9 \%$ for both HAE and PHAF.

Compared to HAE responders, non-responders had more bilateral fitting $(16.9 \%$ versus $24.2 \%$, $P=0.006$ ), but paid for HAs that cost less. Compared with responders for both HAE and PHAF, non-responders were more often fitted with in-ear custom HA, but paid for HAs that cost less (Table 2). No other significant difference was observed. Based on these results, we expect non-responder bias to be minimal.

\section{Baseline hearing disability at HAE}

Subjects reporting more disabilities were older with worse PTA thresholds, and subsequently had higher daily usage (Table 3 ).

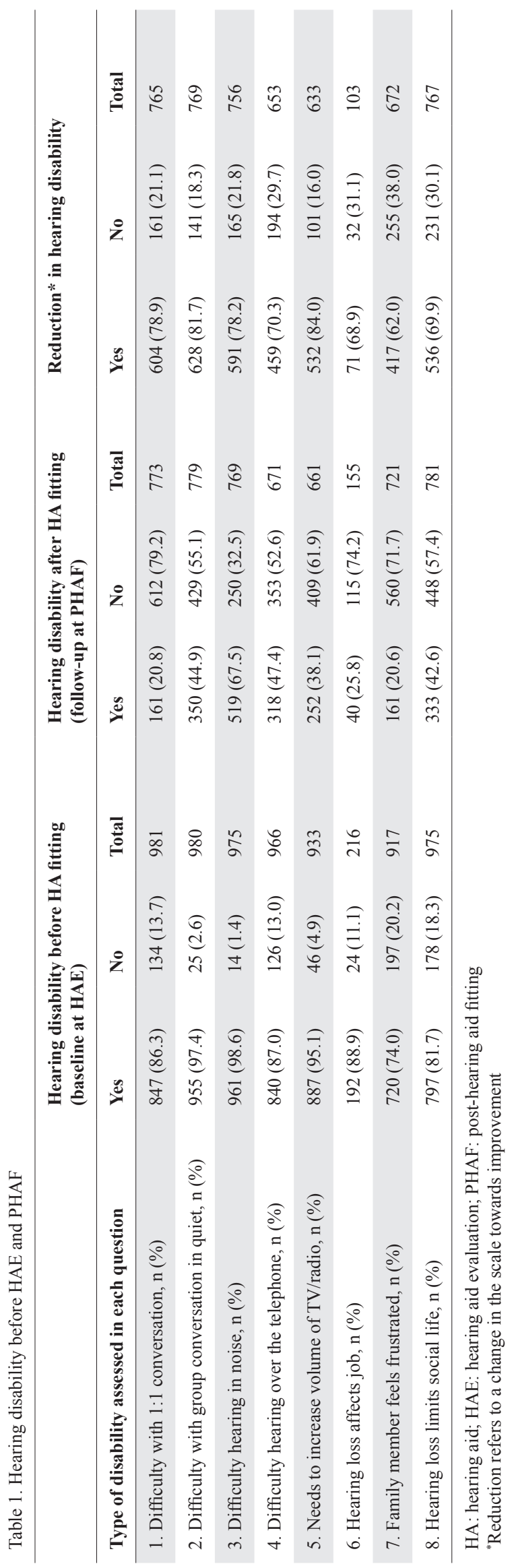

Copyright (C) 2020 Annals, Academy of Medicine, Singapore 


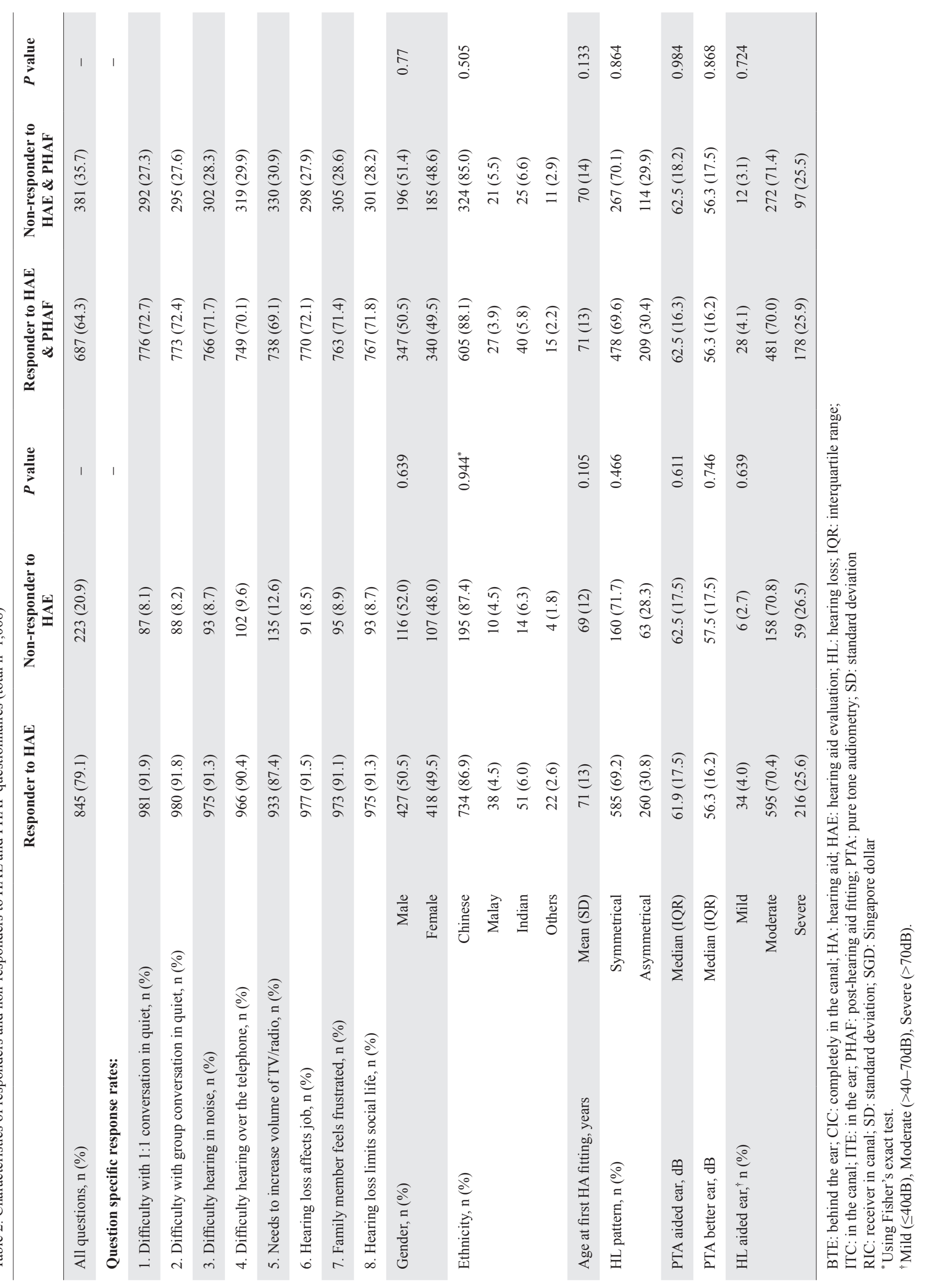




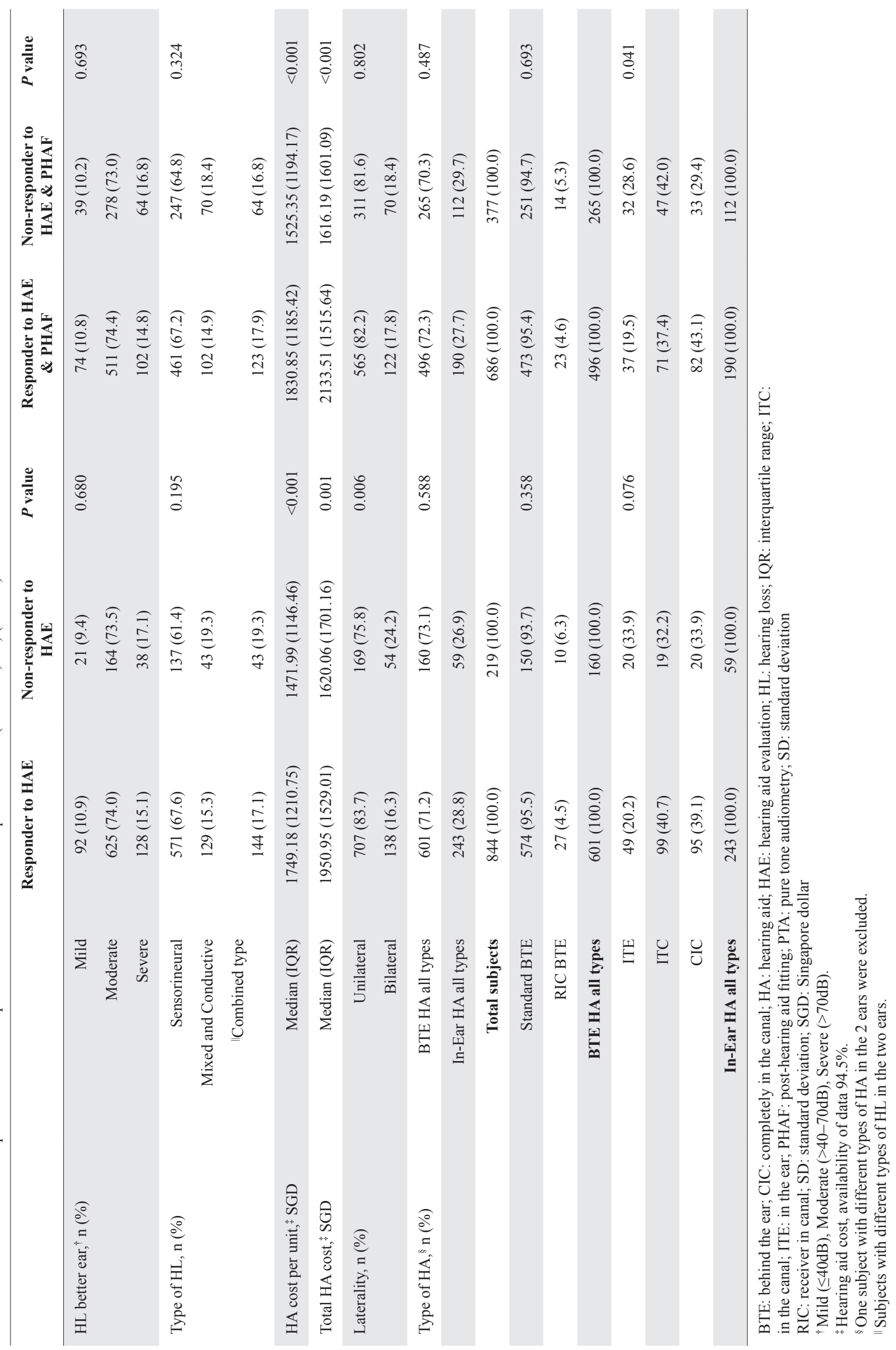




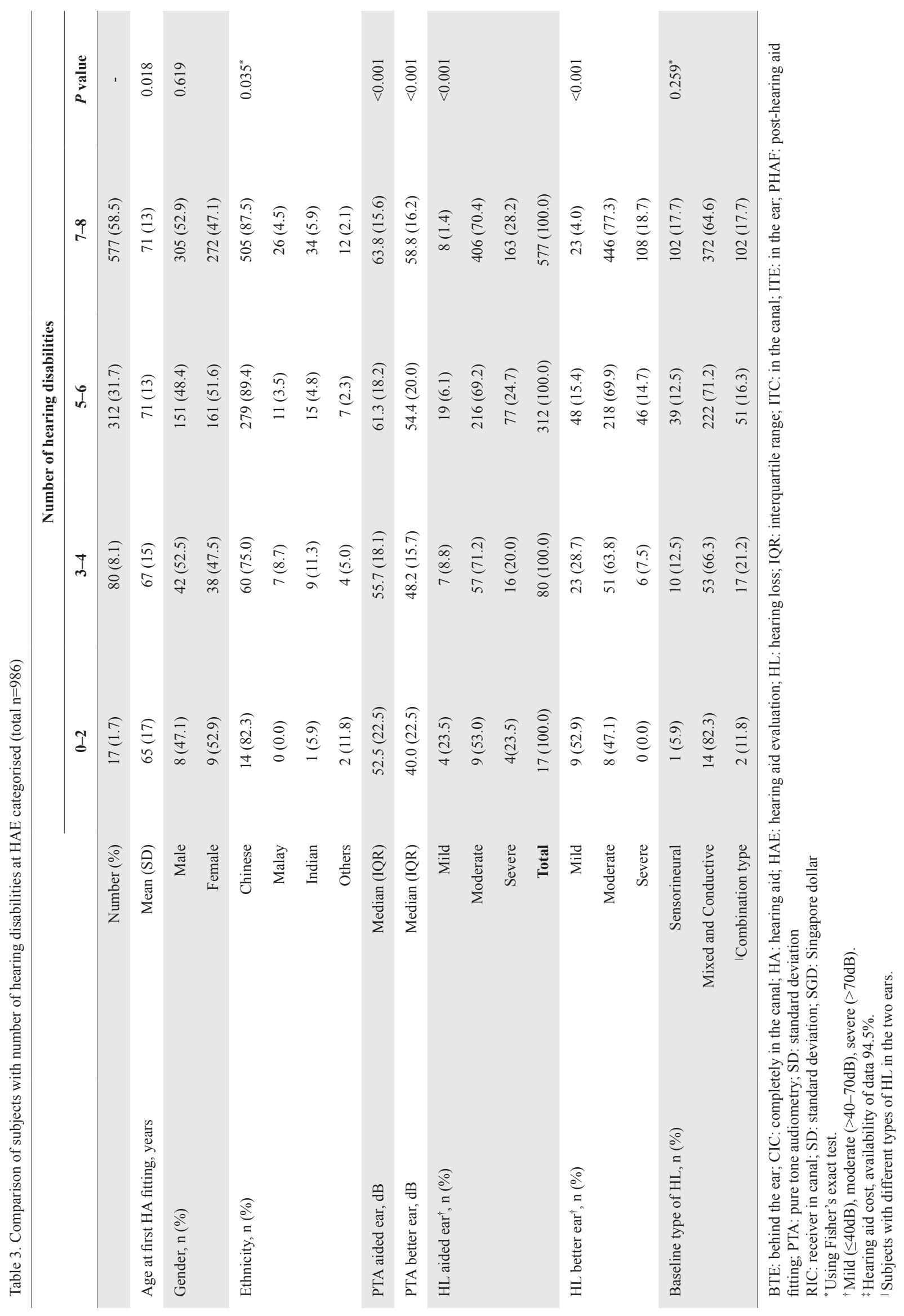




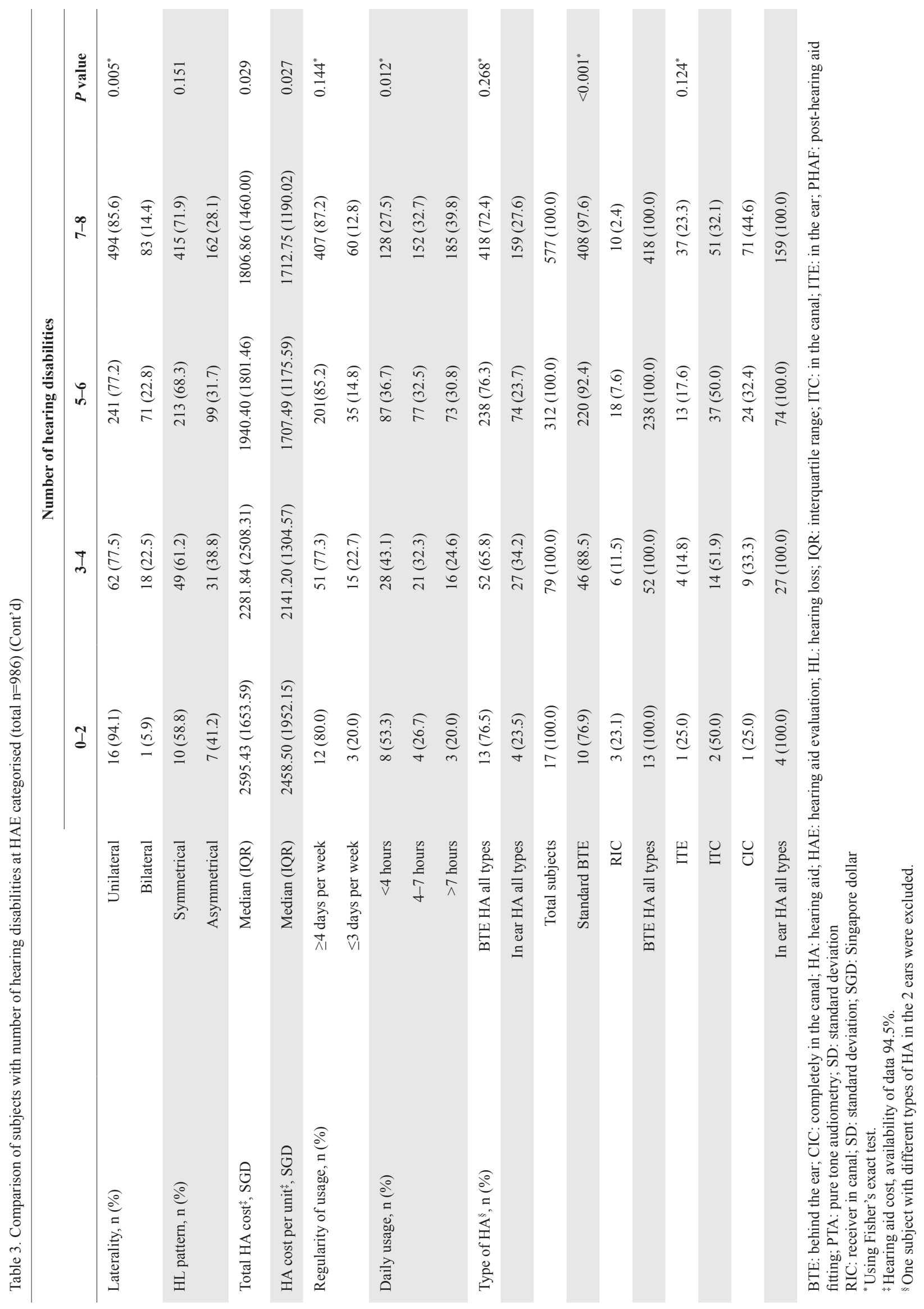


In multivariable models, after adjusting for age, sex, laterality, and aided ear PTA, having worse PTA thresholds of the better ear (AOR $=1.06,95 \%$ CI 1.04-1.08, $P<0.001)$ and being ethnically Chinese compared to Malay $(\mathrm{AOR}=3.04,95 \%$ CI $1.21-7.64, P=0.018)$ were associated with subjects reporting $\geq 5$ compared to $\leq 4$ disabilities at baseline (Table 4).

\section{Reduction in hearing disability at PHAF}

A reduction in $\geq 5$ disabilities was reported by $67.5 \%$ of the subjects at PHAF. Subjects with worse better ear PTA thresholds and who used their HAs more frequently reported reduction of more disabilities.

In multivariable models, after adjusting for age, sex, ethnicity, HL symmetry, aided ear PTA, regularity of usage (self-reported), and type of HA, worse better ear PTA thresholds at baseline (AOR $=1.03,95 \%$ CI 1.01-1.05, $P<0.001)$, sensorineural HL compared to combination type of $\mathrm{HL}(\mathrm{AOR}=1.70,95 \%$ CI $1.02-2.83, P=0.041)$, and daily HA usage for 4-7 hours (AOR $=1.86,95 \%$ CI $1.24-2.80, P=0.003$ ) and $>7$ hours $(\mathrm{AOR}=2.30,95 \%$ CI $1.51-3.52, P<0.001)$ compared to $<4$ hours were associated with subjects reporting reduction in $\geq 5$ disabilities compared to $\leq 4$ disabilities at PHAF (Table 5).

Subjects who reported reduction in 'difficulty with $1: 1$ conversation in quiet' and 'hearing loss limits social life' were more likely to use HA $>7$ hours daily, while those who reported reduction in 'family member feels frustrated' were more likely to use $\mathrm{HA} \geq 4$ days per week.

\section{Discussion}

Compared to Malay subjects, Chinese subjects were more likely to report $\geq 5$ disabilities at HAE. This was despite Malay subjects being more likely to present with more severe HL $(>55 \mathrm{~dB}) \cdot{ }^{26}$ Perception of hearing disability is influenced by the socio-cultural norms of different ethnic groups. Within Singapore, compared to Chinese subjects, Malay subjects had lower odds of reporting poor health despite having higher comorbidities. ${ }^{27,28}$

HAs were self-funded during the study period. Subjects with fewer hearing disabilities paid more for HAs, tended to be younger, more likely to be employed and have better insight into their condition, and also preferred receiver in canal type HAs, which were more expensive. ${ }^{26}$

At the time of the study, HHIE was yet to be validated for use in the Singapore population. Earlier attempts at using HHIE found some questions to be difficult and irrelevant within the socio-cultural context of
Singapore. For example, many subjects would answer 'no' to 'do you feel handicapped by a hearing problem?' despite reporting significant difficulties elsewhere in the questionnaire as the word 'handicap' may potentially carry a negative connotation. Another question, 'does a hearing problem cause you to attend religious services less often than you would like?' may not be relevant to certain subjects. A study on 338 elderly Singapore residents $(92.5 \%$ of whom were non-HA users) found poor correlation between HHIE scores and severity of HL. ${ }^{29}$

However, our HA user subjects showed a good linear correlation between the number of self-reported hearing disabilities at baseline and better ear PTA thresholds. The 10 HHIE questions focus mainly on emotional and social difficulties, ${ }^{29}$ whereas our 8 questions directly assess various situational hearing disabilities.

Worse better ear PTA thresholds, sensorineural HL, and higher daily HA use were independently associated with subjects reporting more reduction in hearing disabilities. European and Australian cohorts have also reported positive correlations between hours of HA use and HA satisfaction..$^{21,23,30}$ This association is not surprising, but causality cannot be determined from our study. Causality for whether subjects used HAs more because of perceived reductions in hearing disabilities or whether increased HA use led to reduction in hearing disabilities can be further explored.

A Taiwanese study found that only $21.4 \%$ of 555 subjects with disabling HL ( $>40 \mathrm{~dB}$ HL of the better ear) reported themselves as hearing handicapped, using a score of $\geq 10$ as the cut-off on the HHIE-Screening. ${ }^{31}$ While essential from an epidemiological viewpoint, the binary 'yes/no' classification of hearing disability has limited usefulness in managing individual patients. Patients' understanding of HL needs to move beyond the 'deaf' versus 'not deaf' dichotomy.

Focusing on patients' self-reported disability and severity will help them understand the continuum of disability. This personalised education and counselling can help patients come to terms with the negative impact HL may have on their quality of life. While hearing disability is related to the severity of HL, ${ }^{32}$ patients' willingness to wear HAs is poorly predicted by the severity of their HL. ${ }^{33}$ Instead, patients who were functionally independent were more accepting of HAs, as they were more likely to feel affected by their hearing disability and handicap. ${ }^{33}$

The results of this study can also help in managing patients' expectations about HAs. For example, for 
Table 4. Unadjusted and adjusted OR and 95\% CI for subjects reporting $\geq 5$ disabilities compared to $\leq 4$ disabilities at $\mathrm{HAE}$

\begin{tabular}{|c|c|c|c|}
\hline \multirow{2}{*}{ Variable } & Unadjusted & Adjusted $^{*}$ & \multirow{2}{*}{$P$ value ${ }^{\dagger}$} \\
\hline & OR $(95 \% \mathrm{CI})$ & OR $(95 \% \mathrm{CI})$ & \\
\hline \multicolumn{4}{|l|}{ Gender } \\
\hline Male & 1.00 & 1.00 & \\
\hline Female & $1.01(0.66-1.53)$ & $1.07(0.68-1.67)$ & 0.763 \\
\hline Age at first fitting (years) & $1.02(1.00-1.03)$ & $1.00(0.98-1.01)$ & 0.985 \\
\hline \multicolumn{4}{|l|}{ Ethnic group } \\
\hline Malay & 1.00 & 1.00 & \\
\hline Chinese & $2.00(0.86-4.65)$ & $3.04(1.21-7.64)$ & 0.018 \\
\hline Indian & $0.92(0.32-2.66)$ & $1.51(0.47-4.81)$ & 0.479 \\
\hline \multicolumn{4}{|l|}{ Laterality } \\
\hline Unilateral & 1.00 & 1.00 & \\
\hline Bilateral & $1.16(0.68-1.97)$ & $1.22(0.69-2.15)$ & 0.492 \\
\hline Better ear PTA (dB) & $1.06(1.05-1.08)$ & $1.06(1.04-1.08)$ & $<0.001$ \\
\hline Aided ear PTA (dB) & $1.03(1.01-1.05)$ & $1.00(0.98-1.01)$ & 0.876 \\
\hline
\end{tabular}

CI: confidence intervals; HAE: hearing aid evaluation; OR: odds ratios; PTA: pure tone audiogram

" Multivariable logistic regression, adjusting for factors shown in table.

${ }^{\dagger} P$ value shown is for adjusted odds ratios.

'difficulty hearing in noise', while $67.5 \%$ of subjects still had this disability after HA fitting, $78.2 \%$ of these subjects had reported a reduction in this disability. Hence, the focus should be on disability reduction rather than complete resolution.

Several study limitations should be considered when drawing conclusions from this study. Factors which could influence subjects' decision for HA use, such as socio-economic status, level of education, concern about cosmesis, and willingness to spend on HAs were not collected. The non-responders in our study also included subjects with dementia, who were unable to answer the questions.

Owing to the retrospective nature of the study, the possible causes of baseline disability cannot be assessed. However, reasonable conclusions can be drawn regarding reduction of disability since the determinants were collected before such reduction. We were unable to examine whether further reduction in disability occurred beyond the first few months after HA fitting as further administration of the questionnaire during subsequent visits was not part of our care protocol. A previous study found no significant changes over time $^{35}$ but the results have not been validated in a
Singapore setting. A prospective study with mid- to long-term follow-up (6 months to over 2 years) would better capture the improvement in disability after HA optimisation. It would be beneficial to study the influence of different fitting formulas and amplification settings on disability reduction in a prospective study.

Despite the above limitations, the study has important strengths. After careful and meticulous extraction, only $10(0.9 \%)$ subjects were excluded from the study due to missing data. A good response rate to the questionnaires (79\% for baseline, and 64\% for both baseline and follow-up) and the absence of detectable responder bias improves the generalisability of the results.

The response scale captured the level of individual hearing disability in finer granularity compared to most earlier studies, allowing a more precise understanding in disability reduction after HA fitting.

As our audiology unit sees one of the largest numbers of HL patients in Singapore, we are confident the study population was representative of the Singapore population. The findings of this study, particularly the ethnic differences in hearing disability and daily usage as a predictor of improvement in disability, have important implications for practice and policy. 
Table 5. Unadjusted and adjusted OR and 95\% CI for subjects reporting reduction in 5 or more disabilities compared to 4 or fewer disabilities at PHAF follow-up

\begin{tabular}{|c|c|c|c|}
\hline \multirow{2}{*}{ Variable } & Unadjusted & Adjusted $^{*}$ & \multirow{2}{*}{$P$ value ${ }^{\dagger}$} \\
\hline & OR $(95 \%$ CI $)$ & OR $(95 \% \mathrm{CI})$ & \\
\hline \multicolumn{4}{|l|}{ Gender } \\
\hline Male & 1.00 & 1.00 & \\
\hline Female & $1.00(0.73-1.35)$ & $1.04(0.75-1.44)$ & 0.808 \\
\hline Age at first fitting (years) & $1.01(1.00-1.02)$ & $1.00(0.99-1.02)$ & 0.155 \\
\hline \multicolumn{4}{|l|}{ Ethnic group } \\
\hline Other & 1.00 & 1.00 & \\
\hline Chinese & $1.42(0.53-3.79)$ & $1.36(0.47-3.95)$ & 0.567 \\
\hline Indian & $2.18(0.62-7.64)$ & $2.07(0.53-8.10)$ & 0.293 \\
\hline \multicolumn{4}{|l|}{ Symmetry } \\
\hline Asymmetrical & 1.00 & 1.00 & \\
\hline Symmetrical & $1.30(0.94-1.81)$ & $0.79(0.50-1.23)$ & 0.298 \\
\hline Better ear PTA (dB) & $1.03(1.01-1.04)$ & $1.03(1.01-1.05)$ & $<0.001$ \\
\hline Aided ear PTA (dB) & $1.01(0.99-1.02)$ & $0.98(0.96-1.00)$ & 0.085 \\
\hline \multicolumn{4}{|l|}{ Baseline type of HL } \\
\hline Combination type & 1 & 1 & \\
\hline Mixed and Conductive & $2.03(1.17-3.52)$ & $1.70(0.91-3.20)$ & 0.095 \\
\hline Sensorineural & $1.44(0.97-2.12)$ & $1.70(1.02-2.83)$ & 0.041 \\
\hline \multicolumn{4}{|l|}{ Regularity of Usage } \\
\hline$\leq 3$ days per week & 1 & 1 & \\
\hline$\geq 4$ days per week & $1.93(1.26-2.95)$ & $1.30(0.80-2.10)$ & 0.282 \\
\hline \multicolumn{4}{|l|}{ Daily usage } \\
\hline$<4$ hours & 1.00 & 1.00 & \\
\hline $4-7$ hours & $1.84(1.26-2.68)$ & $1.86(1.24-2.80)$ & 0.003 \\
\hline$>7$ hours & $2.50(1.71-3.66)$ & $2.30(1.51-3.52)$ & $<0.001$ \\
\hline \multicolumn{4}{|l|}{ Type of HA } \\
\hline In ear HA & 1.00 & 1.00 & \\
\hline Behind the ear HA & $1.27(0.90-1.79)$ & $1.06(0.72-1.55)$ & 0.750 \\
\hline
\end{tabular}

CI: confidence intervals; HA: hearing aid; HL: hearing loss; OR: odds ratios; PHAF: post-hearing aid fitting; PTA: pure tone audiogram

${ }^{*}$ Multivariable logistic regression, adjusting for factors shown in table.

${ }^{\dagger} P$ value shown is for adjusted odds ratios.

* Subjects with different types of HL in the two ears.

\section{Conclusion}

In a sizeable consecutive cohort of first-time HA users in Singapore, we found baseline self-reported hearing disability to be high, with ethnicity and better ear PTA thresholds to be independently associated with disability. Higher daily HA usage, and worse better ear PTA thresholds were associated with reduction in disability after HA fitting. 
Patients with worse hearing and more baseline hearing disability should be reassured that they are more likely to be wearing their HAs, and more likely to report hearing disability reduction. Conversely, patients who fail to report improvement should be counselled to use their HA more frequently.

Counselling patients on the benefits of HL rehabilitation could focus on hearing disability rather than PTA thresholds. The management of patients' expectations could focus on reducing disability rather than eliminating disability.

\section{Acknowledgements}

The authors would like to express appreciation and gratitude to Research Assistants Nuramirah Syed Abdul Rauf, Agnes Chew and Chua Xinning; Biostatisticians Sun Bing and Tang Xin; and Medical Writer Safyya Mohamed Ali. This work was supported by the National Healthcare Group Clinician Scientist Career Scheme under grant CSCS 2013/00325.

\section{REFERENCES}

1. World Health Organization. Deafness and hearing loss, 1 March 2020. Available at: http://www.who.int/mediacentre/factsheets/fs300/en/. Accessed on 27 July 2020.

2. Vos T, Abajobir AA, Abate $\mathrm{KH}$, et al. Global, regional, and national incidence, prevalence, and years lived with disability for 328 diseases and injuries for 195 countries, 1990-2016: a systematic analysis for the Global Burden of Disease Study 2016. Lancet 2017;390:1211-59.

3. World Health Organization. The ICF: An overview, 2010. Available at: https://www.cdc.gov/nchs/data/icd/icfoverview_finalforwho10sept. pdf. Accessed on 27 July 2020.

4. Qi S, Mitchell RE. Large-scale academic achievement testing of deaf and hard-of-hearing students: past, present, and future. J Deaf Stud Deaf Educ 2012;17:1-18.

5. Mohr PE, Feldman JJ, Dunbar JL, et al. The societal costs of severe to profound hearing loss in the United States. Int J Technol Assess Health Care 2000;16:1120-35.

6. Joore MA, Brunenberg DE, Chenault MN, et al. Societal effects of hearing aid fitting among the moderately hearing impaired. Int $\mathrm{J}$ Audiol 2003;42:152-60.

7. Hallam R, Ashton P, Sherbourne K, et al. Persons with acquired profound hearing loss (APHL): how do they and their families adapt to the challenge? Health (London) 2008;12:369-88.

8. Kamil RJ, Lin FR. The effects of hearing impairment in older adults on communication partners: a systematic review. J Am Acad Audiol 2015;26:155-82.

9. Stephens D, Hétu R. Impairment, disability and handicap in audiology: towards a consensus. Int J Audiol 1991;30:185-200.

10. Dawes P, Emsley R, Cruickshanks KJ, et al. Hearing loss and cognition: the role of hearing AIDS, social isolation and depression. PLoS One 2015;10:1-9.
11. Mener DJ, Betz J, Genther DJ, et al. Hearing loss and depression in older adults. J Am Geriatr Soc 2013;61:1627-9.

12. Stephens SD, Callaghan DE, Hogan S, et al. Hearing disability in people aged 50-65: effectiveness and acceptability of rehabilitative intervention. BMJ 1990;300:508-11.

13. Lee JC, Danker AN, Wong YH, et al. Hearing Loss amongst the Elderly in a Southeast Asian Population - A Community-based Study. Ann Acad Med Singap 2017;46:145-54

14. Joore MA, Van Der Stel H, Peters HJ, et al. The cost-effectiveness of hearing-aid fitting in the Netherlands. Arch Otolaryngol Head Neck Surg 2003;129:297-304.

15. Knudsen LV, Oberg M, Nielsen C, et al. Factors influencing help seeking, hearing aid uptake, hearing aid use and satisfaction with hearing aids: a review of the literature. Trends Amplif 2010;14:127-54.

16. World Health Organization. WHO global estimates on prevalence of hearing loss, 2012. Available at: http://www.who.int/pbd/deafness/ WHO_GE_HL.pdf?ua=1. Accessed on 27 July 2020.

17. Ministry of Health, Singapore. National health survey 2010, 2011. Available at: https://www.moh.gov.sg/docs/librariesprovider5/ resources-statistics/reports/nhs2010---low-res.pdf. Accessed on 27 July 2020 .

18. European Hearing Instrument Manufacturers Association (EHIMA). EHIMA - European Hearing Instrument Manufacturers Association, JapanTrak 2012, 2012. Available at: http://www.ehima. com/wp-content/uploads/2014/03/protected japantrak 2012.pdf. Accessed on 27 July 2020.

19. Chien W, Lin FR. Prevalence of hearing aid use among older adults in the United States. Arch Intern Med 2012;172:292-3.

20. Chang HP, Chou P. Presbycusis among older Chinese people in Taipei, Taiwan: a community-based study. Int J Audiol 2009;46:738-45.

21. European Hearing Instrument Manufacturers Association (EHIMA). EHIMA - European Hearing Instrument Manufacturers Association, EuroTrak UK 2009, 2009. Available at: http:/www.ehima. com/wp-content/uploads/2014/03/protected eurotrak 2009 uk pr.pdf. Accessed on 27 July 2020.

22. Ho EC, Zhang H, Ong WMW, et al. Hearing impairment and hearing aid usage in Singapore. Int J Audiol 2018;57:291-301.

23. European Hearing Instrument Manufacturers Association (EHIMA). EHIMA - European Hearing Instrument Manufacturers Association, EuroTrak Switzerland 2012,2012. Available at: http://www. ehima.com/wp-content/uploads/2014/03/protected_eurotrak_2012 switzerland.pdf. Accessed on 27 July 2020.

24. European Hearing Instrument Manufacturers Association (EHIMA). EHIMA - European Hearing Instrument Manufacturers Association, EuroTrak Germany 2009, 2009. Available at: http://www. ehima.com/wp-content/uploads/2014/03/protected_eurotrak 2009 ger_pr_.pdf. Accessed on 27 July 2020.

25. Dillon H, James A, Ginis J. Client Orientated Scale of Improvement (COSI) and its relationship to several other measures of benefit and satisfaction provided by hearing aids. J Am Acad Audiol 1997;8:27-43.

26. Ho EC, Ong WMW, Li K, et al. Factors influencing degree of hearing loss at presentation, hearing aid choice, and usage in first time hearing aid users in Singapore. Int J Audiol 2018;57:776-83.

27. Lim WY, Ma S, Heng D, et al. Gender, ethnicity, health behaviour \& self-rated health in Singapore. BMC Public Health 2007;7:184.

28. George PP, Heng BH, De Castro Molina JA, et al. Self-reported chronic diseases and health status and health service utilization - 
results from a community health survey in Singapore. Int J Equity Health 2012;11:44.

29. Weinstein BE, Ventry IM. Audiometric correlates of the Hearing Handicap Inventory for the elderly. J Speech Hear Disord 1983; 48:379-84.

30. Hickson L, Timm M, Worrall L, et al., Hearing aid fitting: Outcomes for older adults. Aust J Audiol 1999;21:9-21.

31. Chang HP, Ho CY, Chou P. The factors associated with a selfperceived hearing handicap in elderly people with hearing impairment-results from a community-based study. Ear Hear 2009;30:576-83.
32. Metselaar M, Maat B, Krijnen P, et al. Self-reported disability and handicap after hearing aid fitting and benefit of hearing aids: comparison of fitting procedures, degree of hearing loss, experience with hearing aids and uni- and bilateral fittings. Eur Arch Otorhinolaryngol 2009;266:907-17

33. Wu HY, Chin JJ, Tong HM. Screening for hearing impairment in a cohort of elderly patients attending a hospital geriatric medicine service. Singapore Med J 2004;45:79-84.

34. Munro KJ, Lutman ME. Self-reported outcome in new hearing aid users over a 24-week post-fitting period. Int J Audiol 2013; 43:555-62. 